\title{
Quantum Dots as Cellular Probes for Light and Electron Microscopy
}

\author{
Thomas J. Deerinck, Ben N.G. Giepmans and Mark H. Ellisman
}

The National Center for Microscopy and Imaging Research, University of California, San Diego, La Jolla, CA, 92093-0608

Fluorescent semiconductor nanocrystals, also referred to as Quantum dots, are becoming increasingly popular as cellular probes for light microscopic imaging due to their unique optical and physical properties. These include high fluorescent quantum yields, resistance to photobleaching, and narrow band fluorescence emission. They can be synthesized in a variety of distinct shapes and sizes in order to tune their fluorescence emission over the visible spectrum, and unlike commonly used organic fluorophores such as fluorescein and rhodamine, they all exhibit similarly large absorption cross sections, making them well suited for simultaneous excitation from a single light source (1). Additionally, various Quantum dots have sufficient electron density and size diversity to be easily distinguished by electron microscopy, making multiple labeling of different proteins possible (2). While much attention has been focused on their application to live-cell imaging, the utility of Quantum dot-secondary antibody conjugates for protein labeling in fixed cells and tissues has not yet been fully characterized and exploited.

We have been testing six types of commercially available Quantum dot F(ab')2 anti-IgG conjugates exhibiting 525, 565, 585, 605, 655 and $705 \mathrm{~nm}$ fluorescence. When applied to a thin carbon film, air dried, and imaged by electron microscopy they each possess a distinct size, with a shape transition from round to rod-shaped occurring between 585 and $605 \mathrm{~nm}$. In general, the larger the quantum dot, the longer the fluorescence emission spectra (3). Antibody conjugates of these probes exhibit modest but significant penetration ( $>5$ microns in many cases) into aldehyde fixed and mildly permeablized cells and tissues, making them suitable for pre-embedding labeling methods. Although no universal protocol exists for immunolabeling all antigens with sufficient ultrastructural preservation for electron microscopy, we have established some general criteria to allow successful multiple labeling of a variety of diverse proteins for both light and electron microscopy.

Cultured cells or tissues are fixed with 2-4 \% formaldehyde (from paraformaldehyde), in some instances with the addition of $0.05-0.5 \%$ glutaraldehyde. Permeablization with low concentrations of Triton X-100 or saponin is accomplished at $4^{\circ} \mathrm{C}$. For double labeling we employed 565 and $655 \mathrm{~nm}$ Quantum dots and for triple labeling we used 525, 565 (or 585), and $655 \mathrm{~nm}$ Quantum dots, though other combinations are possible. One of the main attractions of using Quantum dots is that they allow for efficient trouble-shooting and optimization of various antibody-antigen dependent labeling parameters by light microscopy prior to proceeding with the more time consuming and laborious preparation for electron microscopy.

\section{References}

[1] W.C. Chan and S. Nie (1998) Science 281, 2016-2018.

[2] R. Nisman et al. (2004) J Histochem Cytochem. 52(1):13-8.

[3] W.C. Chan et al. (2002) Curr. Opin. Biotechnol. 13(1), 40-46.

[4] This work was supported by NIH grants NS 14718 and NCRR RR04050. 

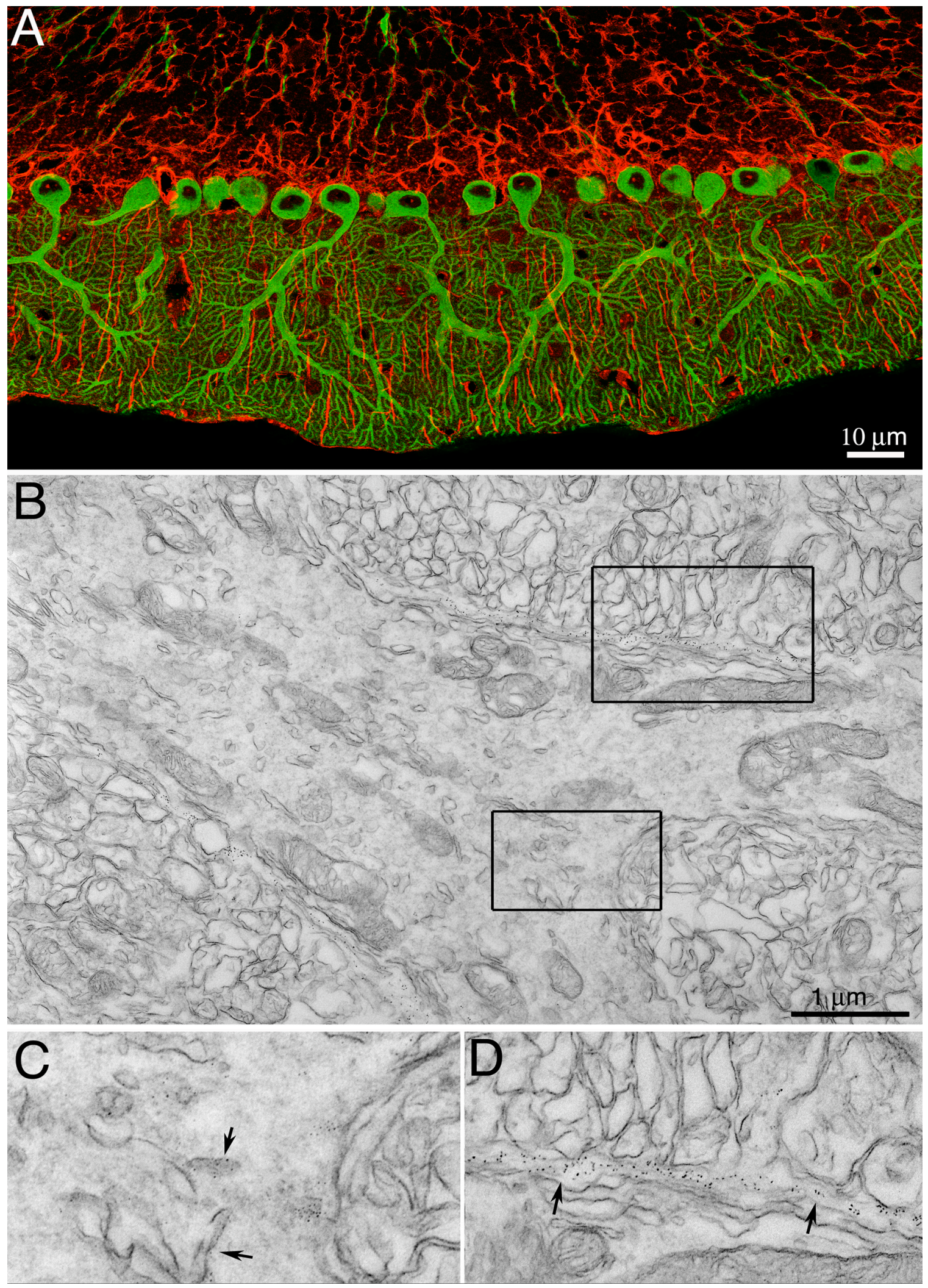

Figure 1. Mouse cerebellum double immunolabeled for the IP3 receptor (QD 565) and GFAP (QD 655, red). A) Confocal image showing prominent labeling of Purkinje cells (green fluorescence) and glial cells (red fluorescence). B) Electron micrographs showing QD 565 labeling of the ER in a large Purkinje cell dendrite (see enlarged area in C) and QD 655 labeling in a nearby glial cell process (see enlarged area in D). 